\title{
Ações integradas no gerenciamento de resíduos e recuperação ambiental na Faculdade de Formação de Professores da UERJ, São Gonçalo, RJ
}

Integrated actions on residues management and environmental recovery in the Teachers College (FFP) of Rio de Janeiro State University (UERJ)

\section{Introdução}

Desde o início da Revolução Industrial até os dias atuais, foi notório o crescimento populacional humano de forma acelerada e o aumento dos diversos tipos de resíduos gerados como subprodutos de suas atividades. Tais resíduos ultrapassam a capacidade real de retornar ao estado natural de excelência, gerando desequilíbrios em seus ciclos originais. Grandes fluxos de elementos artificiais em altas concentrações, muitos deles tóxicos e nocivos à vida na biosfera, são depositados a todo o momento em regiões em que o seu subsistema gira em torno da própria dinâmica da natureza ${ }^{1}$. Este fluxo de deposição dos rejeitos acaba voltando ao ciclo de vida dos seres humanos sob formas de poluição, radiação, contaminação, chuva ácida, entre outras².

De acordo com os dados do IBGE em 2000, o lixo produzido diariamente no Brasil chegava a 125.281 toneladas, sendo que $47,1 \%$ era destinado a aterros sanitários, $22,3 \%$ a aterros controlados e $30,5 \%$ a lixões. Porém, esses mesmos resíduos não são percebidos como uma significativa preocupação ambiental por nossa sociedade. A solução deste problema quase sempre é adiada até ocorrerem ameaças, iniquidades e conflitos ambientais mais graves às pessoas que estão diretamente ligadas a esses contextos, tais como as populações que habitam o entorno de áreas degradadas, como exemplo, aquelas em que a deposição de resíduos se apresenta potencial e efetivamente com altos níveis de poluição e contaminação ${ }^{1}$. Tais constatações nos remetem à necessidade de uma política e atitudes que promovam a Educação Ambiental.

A utilização do termo "Educação Ambiental" remonta a 1965. Porém, como resultado das recomendações da Conferência das Nações Unidas sobre Ambiente Humano, de 1972 em
Marcelo Guerra Santos', Gabriela Rodrigues da Silva², Maria Luiza Costa Azevedo ${ }^{3}$, Mariana Macêdo Barcellos ${ }^{4}$, Mariana Manhã Sérgio ${ }^{5}$, Renan de França Souza ${ }^{6}$, Ana Angélica Monteiro de Barros ${ }^{7}$, Douglas de Souza Pimentel ${ }^{8}$, Luiz José Soares Pinto ${ }^{9}$, Maria Cristina Ferreira dos Santos ${ }^{10}$, Regina Rodrigues Lisbôa Mendes ${ }^{11}$, Ricardo Tadeu Santori ${ }^{12}$

\section{Resumo}

O presente trabalho teve como objetivos apresentar uma análise qualitativa e quantitativa dos resíduos produzidos na Faculdade de Formação de Professores (FFP) da Universidade do Estado do Rio de Janeiro (UERJ) e fazer o diagnóstico do gerenciamento destes resíduos; proposição de medidas mitigadoras e realização de atividades extensionistas de Educação Ambiental em escolas de São Gonçalo. O diagnóstico do gerenciamento dos resíduos na FFP foi feito através de entrevistas estruturadas e a análise qualitativa e quantitativa por meio da pesagem e identificação visual durante cinco semanas. Atividades de observação de aves para sensibilização ambiental foram realizadas na área de reflorestamento da FFP. A análise quantitativa mostrou uma produção per capita de resíduos sólidos de 26,46g/usuário por dia. Os resultados indicam a necessidade de uma política mais integrada de gerenciamento de resíduos que não se resuma somente à limpeza das dependências da Unidade. As ações mitigadoras no presente são: a reutilização do papel na produção de blocos de anotações, compostagem dos resíduos vegetais e reciclagem do óleo de cozinha usado na cantina para fabricação de sabão. Na área de reflorestamento da FFP já foram observadas 41 espécies de aves. As ações futuras incluem a elaboração de um plano de gerenciamento de resíduos químicos, redução dos recicláveis, reflorestamento de parte da área e produção de um vídeo temático.

Palavras-chaves: Ações Educativas; Gerenciamento de Resíduos; Reflorestamento; Observação de aves

\footnotetext{
Área temática: Meio Ambiente Linha da extensão: Questões Ambientais

Docente e Procientista. UERJ. E-mail: marceloguerrasantos@gmail.com 2 Aluna de graduação. UERJ. E-mail: gabri_cbjr@hotmail.com

Aluna de graduação. UERJ. E-mail: marialuizaazevedoffp@gmail.com ${ }^{4}$ Aluna de graduação. UERJ. E-mail: mari.bio09@hotmail.com

Aluna de graduação. UERJ. E-mail: marianamanha@yahoo.com

Aluno de graduação. UERJ. E-mail: renan1604@hotmail.com

7 Docente. UERJ. E-mail: anaangbarros@gmail.com

Docente. UERJ e UFF. E-mail: douglasgeia@gmail.com

Docente. UERJ. E-mail: Ijspinto@gmail.com

${ }^{10}$ Docente. UERJ. E-mail: mcfs@ueri.br

Docente. UERJ. E-mail: rrlmendes@ig com.br

2 Docente e Procientista. UERJ. E-mail: rsantori.uerj@gmail.com
} 
Estocolmo, a partir de 1975 torna-se um objeto de análise específica em Educação, com a realização do I Seminário Internacional de Educação Ambiental em Belgrado ${ }^{3}$. Esse seminário também estabeleceu referências para as diretrizes norteadoras das atividades de Educação Ambiental na Conferência Intergovernamental sobre Educação Ambiental, realizada em Tbilisi, na Geórgia em 1977, e que hoje são aceitas internacionalmente. Logo, a Educação Ambiental relaciona-se às questões da conservação no âmbito socioeconômico, no campo da política e da cidadania ${ }^{4,5}$.

No Brasil, esse processo de discussão também começa a partir da década de 1970, porém, só ganha maior dimensão pública na década de 1980, consolidando-se na Constituição Federal de 1988, que estabelece a promoção da Educação Ambiental como competência do Poder Público. Em 1999 é publicada a lei que institui a "Política Nacional de Educação Ambiental" (PNEA - lei $n^{\circ} 9.795$ de 27 de abril de 1999) que reforça a visão desse processo educativo acontecendo de forma articulada em caráter formal e não formal ${ }^{6,7}$.

Assim, a Educação Ambiental segue diferentes diretrizes e conceituações. Nessas visões diversas pode-se destacar a importância da capacitação das pessoas para a compreensão crítica do ambiente em um processo contínuo que busca compreender as relações socioambientais, encaradas holisticamente no tempo e no espaço. Essa deve estimular a capacidade de detecção dos sintomas e causas dos problemas ambientais para a busca conjunta da resolução dos mesmos, sob um enfoque interdisciplinar, que incentive a participação de indivíduos e coletividade, sua vivência prática e experiências ${ }^{3}$. Neste contexto é que a Faculdade de Formação de Professores da Universidade do Estado do Rio de Janeiro (FFP/UERJ) se insere com o desafio de promover uma análise dos subprodutos gerados a partir do seu próprio funcionamento, a reflexão crítica sobre os problemas ambientais e a proposição de práticas educativas, assumindo sua responsabilidade maior que é a formação de educadores.

A FFP/UERJ possui uma grande área ${ }^{8}$, que outrora pertenceu à Fazenda do Jacaré, do Barão de São Gonçalo e no século XIX abrangeu $219.000 \mathrm{~m}^{2}$. Parte da fazenda foi adquirida pelo Governo do Estado do Rio de Janeiro em 1915, uma área de $55.000 \mathrm{~m}^{2}$. O casarão de dois andares da fazenda deu lugar ao Patronato de Menores e posteriormente ao Grupo Escolar Barão de São Gonçalo, atualmente desativado?. Hoje essa relí- quia histórica encontra-se totalmente em ruínas. O restante da área da fazenda está ocupada por escolas públicas, como o Colégio Estadual Walter Orlandine, o CIEP Jornalista Wladimir Herzog, o CIEP Prof. Djair Cabral Malheiros, a Escola Estadual Coronel João Tarcísio Bueno, a APAE de São Gonçalo, o Centro de Reabilitação Niuma Goulart Brandão, a Central de Rádio Amadores, a Paróquia de Nossa Senhora Aparecida, conhecida como Igreja do Patronato, além de moradias nos bairros do Patronato e Paraíso ${ }^{10}$.

Uma série de impactos ambientais é observada na região. Esses são caracterizados pela ocupação imobiliária irregular, pela retirada da vegetação original e ocupação por espécies exóticas invasoras, como o capim-colonião (Panicum maximus Jacq.), que dificulta a recuperação ambiental. Além disso, os impactos sociais são representados pela destinação inadequada do lixo e das águas servidas das residências.

Partindo do pressuposto de que o indivíduo também deve ser responsável pelos resíduos que produz e pela sua correta destinação, assim como da inclusão da Educação Ambiental no currículo escolar como tema transversal (Parâmetros Curriculares Nacionais - PCNs), foram realizadas neste trabalho diferentes ações no sentido de melhorar o gerenciamento dos resíduos no campus da FFP, através do projeto de extensão "O futuro é agora: sensibilização de futuros professores na busca por um mundo ambientalmente equilibrado" (maiores informações no blog: http://socioambientalffp.blogspot.com. br). Sendo uma unidade formadora de professores, é importante que os licenciandos tenham contato com práticas que visem à promoção de um ambiente com melhor qualidade de vida. Nesse sentido, o presente trabalho teve como objetivos as seguintes ações: análise qualitativa e quantitativa dos resíduos produzidos nas dependências da FFP; diagnóstico do gerenciamento dos resíduos na FFP; proposição de medidas mitigadoras aos resíduos produzidos na FFP; realização de atividades de Educação Ambiental e promoção de práticas extensionistas com as escolas vizinhas e parceiras da FFP.

\section{Metodologia}

\section{Análise da produção de resíduos sólidos na FFP/UERJ}

A FFP/UERJ apresenta 2.650 alunos, 222 docentes efetivos e 60 técnico-administrativos, 
totalizando uma comunidade de cerca de 3.000 indivíduos ${ }^{11}$.

O diagnóstico do gerenciamento dos resíduos produzidos na FFP foi realizado através de entrevistas estruturadas com os diferentes atores envolvidos diretamente na geração e/ou administração dos resíduos, entre eles o administrador (1), biólogos e técnicos de laboratório (6), funcionários da cantina (2), funcionário da central de cópias (1) e funcionários da limpeza (12). Para facilitar a análise quantitativa e qualitativa, a FFP foi setorizada de acordo com os locais em que os resíduos são produzidos. Assim, os setores determinados foram: cantina, reprografia e prédios/ pátio. Os prédios compreendem as salas de aula, gabinetes, laboratórios, biblioteca e secretarias. Diariamente, durante 5 semanas (março a abril de 2011), os resíduos produzidos foram qualificados e mensurados com uma balança de capacidade de $22 \mathrm{~kg}$. Na avaliação da produção per capita de resíduos sólidos na FFP foi utilizada a fórmula de Oliveira ${ }^{12}$, com modificações.

\author{
$\mathrm{PpcRSU}=\mathrm{P} / \mathrm{N}$ \\ PpcRSU = Produção per capita de resíduos \\ sólidos universitários (g.usuário-1.dia-1) \\ $\mathrm{P}=$ Peso dos resíduos sólidos produzidos \\ $\mathrm{N}$ = número de usuários da Universidade
}

\section{Atividade de observação de aves na FFP}

Entendemos que a observação de aves pode ampliar a percepção ambiental das pessoas, uma vez que ao observar uma ave também atentamos para o ambiente à sua volta. As aves podem ser boas indicadoras da qualidade ambiental e sua observação em áreas urbanas chama nossa atenção para a interdependência entre natureza e sociedade. Desta forma, ao promover esta atividade na área da FFP, procuramos estimular a reflexão sobre a necessidade de repensarmos essa relação, em vista dos prejuízos gerados para ambas as partes com a escolha da manutenção de um sistema de exploração predatória dos ambientes e dos recursos naturais.

Promovemos oficinas de observação de aves em eventos dentro da própria FFP. Nestas oficinas, os participantes foram divididos em no máximo 15 duplas, em que cada dupla recebeu um binóculo (7 X $35 \mathrm{~mm}$ ), uma prancheta, uma caixa de lápis de cor e fichas de observação contendo uma figura inacabada de uma ave hipotética para completar e colorir segundo as características das aves observadas. Os participantes foram orientados, nos locais de observação, por monitores do projeto que os auxiliaram com o uso do material e com os procedimentos para uma boa observação. Após o período de observação, os participantes identificaram as aves observadas comparando as figuras coloridas das fichas com as ilustrações de diferentes publicações de aves, fornecidas pela equipe do projeto ${ }^{13,14,15,16,17,18,19}$. Ao término da identificação, foi ministrada uma palestra pelo coordenador da atividade sobre a importância das aves no ambiente e uma discussão sobre as espécies observadas. As oficinas foram concluídas com a avaliação da atividade pelos participantes.

\section{Resultados e Discussão}

\section{Diagnóstico do gerenciamento dos resíduos produzidos na FFP}

Com relação aos resíduos químicos produzidos pelos laboratórios, a maioria é descartada de maneira inadequada, sendo colocados em recipientes vazios e estocados nos próprios laboratórios ou em espaços improvisados sem nenhum critério de separação. Quando é necessário o descarte de materiais perfurocortantes, eles são colocados em recipientes tipo "descarpack", porém posteriormente são misturados aos resíduos comuns. A ausência de um local adequado ao descarte de produtos químicos produzidos na FFP traz como consequência o risco eminente de acidentes. Segundo os depoimentos dos profissionais que produzem resíduos biológicos, os animais utilizados em aulas práticas não são incinerados, o descarte biológico é armazenado dentro do laboratório e os resíduos microbiológicos passam por um processo de esterilização, sendo depois descartados na lixeira comum. Verificou-se também que a minoria dos profissionais realizou curso de biossegurança em laboratório. Não há na unidade qualquer tipo de gerenciamento para o descarte dos resíduos tanto químicos quanto biológicos, inclusive a presença de animais mortos e produtos tóxicos, como formol, encontrados dentro de lixeiras de uso comum ressaltam esta afirmação. 
Tem-se uma alta estimativa do consumo de copos descartáveis na FFP, aproximadamente, 300 copinhos de café e 200 de água por dia apenas na administração. Tais resíduos não são seletivamente descartados assim como a maioria, a única orientação especial que se tem na unidade é em relação ao destino de lâmpadas fluorescentes, que são apenas separadas dos outros resíduos. Também são produzidos na FFP resíduos vegetais tais como folhas, galhos pequenos das árvores e resíduos de grama que são empilhados para virar húmus. Aproximadamente $40 \%$ destes são jogados no lixo comum e $60 \%$ são descartados no terreno vizinho juntamente com os galhos maiores de árvores. Até o início do desenvolvimento deste projeto não havia prática mitigadora para os resíduos produzidos na FFP.

\section{Análise quantitativa e qualitativa dos resíduos}

A produção per capita de resíduos sólidos nos diferentes setores da FFP e a análise qualitativa é apresentada na tabela 1. Podemos verificar que a cantina é a maior geradora de resíduos: 13,72g/usuário dia. Além dos resíduos sólidos, a cantina ainda utiliza semanalmente uma média de 9,5 litros de óleo de cozinha. No somatório, a FFP/UERJ produz 26,46 g/ usuário dia, média próxima da registrada no campus de São Gabriel, na Universidade Federal do Pampa (UNIPAMPA): 23,24g/usuário dia ${ }^{20}$. A comunidade da FFP/UERJ possuía em 2010 cerca de 3000 indivíduos $^{11}$ enquanto a UNIPAMPA-São Gabriel era de apenas 420 indivíduos em $2008^{20}$. Ou seja, a produção de resíduos na FFP/UERJ foi menor que na UNIPAMPA-São Gabriel. Essas médias são bem inferiores àquelas registradas para os resíduos sólidos urbanos (RSU) e domiciliares (RSD). No município do Rio de Janeiro, por exemplo, a produção de RSU é de $1.424 \mathrm{~g} /$ habitante dia ${ }^{21,22}$. Apesar de não ter sido realizada uma quantificação por categoria dos resíduos produzidos, é notória a maior contribuição dos resíduos orgânicos oriundos de restos alimentares (cantina) e de jardinagem (no pátio) na geração total. Desse modo, é importante canalizar ações para a mitigação através de atividades da compostagem orgânica.

Tabela 1: Análise qualitativa e produção per capita de resíduos sólidos universitários (g.usuário-1.dia-1) nos diferentes setores da FFP/UERJ, no período de março a abril de 2011

\begin{tabular}{lll}
\hline Reprografia & Cantina & Prédios/pátios \\
\hline Papéis & Orgânicos & Resíduos de jardinagem \\
Caixa de papelão & Latas de alumínio & Papéis \\
Plástico & Copos descartáveis & Copos descartáveis \\
& Caixas de madeira & Lâmpadas \\
& Óleo de cozinha & Papel higiênico \\
& & Plásticos \\
\hline 0,74 g/usuário dia & 13,72 g/usuário dia & 11,89 g/usuário dia \\
\hline
\end{tabular}

\section{Ações mitigadoras no presente}

\section{Reutilização do papel da reprografia}

Os papéis descartados na reprografia e/ou por ações administrativas estão sendo reutilizados para produzir blocos de anotações. Esses blocos estão sendo disponibilizados aos alunos frequentadores da biblioteca e, segundo relatos da bibliotecária-chefe tem sido muito utilizados pelos usuários, uma medida simples, mas de grande impacto dentro da comunidade universitária.

\section{Óleo da cantina}

O óleo descartado na cozinha da cantina está sendo utilizado para fabricação de sabão em barra e sabonete líquido, com a intenção de, em um futuro próximo, disponibilizar, nos banheiros da FFP/UERJ, o sabão líquido produzido. Esta ativi- 
dade vem despertando o interesse de diversas escolas parceiras da FFP/UERJ e frequentemente o projeto oferece a oficina "Produzindo o seu próprio sabão: a importância ambiental de reciclar o óleo de cozinha”. Tal oficina desperta a atenção dos participantes principalmente pelo menor gasto de recursos financeiros, pois, com um litro de óleo usado, a receita do sabão líquido, por exemplo, rende aproximadamente 4 litros e o em barra 3 quilos, sendo o valor investido inferior ao preço de venda do sabão no mercado. Em cada apresentação da oficina foram entregues informativos contendo as receitas, além de um roteiro da atividade e advertências em relação ao uso da soda cáustica, uma das substâncias utilizadas durante o procedimento.

Foi realizada uma exposição sobre a fabricação dos sabões em barra e líquido durante o "Bio na Rua”, evento de extensão promovido pelo Centro Acadêmico do curso de Ciências Biológicas em parceria com do Departamento de Ciências (DCIEN). Esse evento tem por objetivo divulgar as atividades realizadas por biólogos e professores na comunidade e ocorre anualmente nas praças do município de São Gonçalo.

\section{Compostagem}

A reciclagem dos resíduos orgânicos é realizada por meio da compostagem. Este processo consiste em amontoar resíduos vegetais e/ou animais e mediante tratamentos químicos ou não, acelerar a sua decomposição. O produto resultante é o que chamamos de composto orgânico. Esse composto estimula a proliferação de microrganismos úteis; melhora as qualidades físicas do solo; aumenta a capacidade de retenção de água e nutrientes; facilita a drenagem e fornece substâncias que estimulam o crescimento das plantas. Os resíduos vegetais oriundos da cantina e das atividades de poda e capina do campus da FFP/UERJ são recolhidos e triturados em um triturador elétrico. Esse material é organizado em leiras de $1 \mathrm{~m}$ de largura e cerca de 0,50m de altura. Todo composto orgânico gerado será utilizado na produção de mudas de plantas medicinais e para o reflorestamento.

\section{Observação de aves}

O reflorestamento que vem sendo realizado na FFP/UERJ desde o ano de 1999 tem contribuído para o aumento da diversidade da avifauna no campus da referida unidade ${ }^{23,24}$. Desse modo, a atividade de observação de aves torna-se bastante interessante como instrumento de Educação Ambiental.

Registramos até o momento uma lista de 41 espécies de aves que ocorrem na FFP/UERJ. A maioria delas são aves de ampla distribuição geográfica, de hábitos alimentares generalistas e espécies tolerantes a ambientes alterados pelo homem. Algumas são comuns em áreas abertas, matas secundárias e capoeiras. Estas informações são condizentes com o tipo de paisagem onde as observamos, já que a FFP/UERJ está situada numa área que era originalmente coberta por Mata Atlântica, que foi totalmente desmatada para dar lugar a diferentes tipos de cultivo, até se tornar o que é hoje; um terreno erodido, com solo compactado, colonizado pelo capim colonião, que sofre com a ação do fogo, despejo irregular de lixo pela população vizinha, pastagem de ruminantes e um processo de ocupação irregular na vizinhança. O trabalho de registro das espécies de aves começou em 2002, sendo contínuo desde então ${ }^{24,25}$.

Após analisar as respostas de 32 participantes das oficinas de observação de aves ao questionário de avaliação da oficina, verificamos que o comentário mais frequentemente feito pelos participantes, ao final da atividade, é sobre como eles nunca haviam percebido o quanto as aves são interessantes. Quase que a totalidade dos participantes $(70 \%)$ nunca tinha parado para observar aves no seu dia a dia. $\mathrm{O}$ aspecto das aves que mais chamou a atenção dos participantes foram suas cores (64\%), o que só é bem percebido para muitas espécies quando são cuidadosamente observadas. Além de conhecerem melhor a biologia das aves, os participantes foram estimulados a desenvolver o senso de observação, disciplina, concentração e sociabilidade, o que mostra que esta atividade pode ser utilizada para a melhoria da percepção ambiental.

A observação das aves apresenta um grande potencial para a Educação Ambiental pelo fato de despertar carisma nas pessoas devido às suas características ${ }^{26}$. À medida que as aves vão sendo mais conhecidas e compreendidas por nós, suas chances de serem preservadas aumentam, pois aprendemos a admirá-las e a valorizá-las, reconhecendo seu papel no ambiente. Preservando as aves, estamos ajudando a preservar os ambientes naturais. Nossa 
ideia é que a continuação do reflorestamento na FFP/UERJ adicione mais recursos ao ambiente e crie condições de sobrevivência para mais espécies, aumentando a diversidade da avifauna. Neste sentido, o monitoramento das espécies poderá ser um bom indicador da qualidade do ambiente. Maiores informações sobre a observação de aves podem ser encontradas no blog do projeto http:// observacaodeavesffp.blogspot.com/.

\section{Ações mitigadoras futuras}

\section{Plano de Gerenciamento de Resíduos Químicos (PGRQ)}

Infelizmente, ainda é prática comum nas instituições universitárias o descarte inadequado da maioria dos resíduos químicos - muitas vezes diretamente nas pias dos laboratórios. Essa prática incorreta impacta negativamente o ambiente. Os resíduos perigosos gerados nos laboratórios de ensino e pesquisa necessitam de mecanismos seguros para a sua passivação e/ou disposição final, já que eles requerem um procedimento de descarte muito distinto daquele dado ao lixo doméstico.

Entre as ações mitigadoras a serem realizadas na FFP/UERJ, inclui-se a elaboração de um Plano de Gerenciamento de Resíduos Químicos, que permitirá apontar e descrever as ações relativas ao seu manejo.

Ao ser implantado, o programa precisa considerar dois tipos de resíduos: o ativo (gerado continuamente) e o passivo, que compreende todos os resíduos estocados, muitas vezes não caracterizados, aguardando destinação final. $\mathrm{O}$ resíduo ativo é a principal preocupação de qualquer programa de gerenciamento. $O$ passivo inclui restos reacionais, resíduos sólidos e frascos de reagentes lacrados, mas sem rótulos ${ }^{27}$. A FFP/UERJ tem resíduo passivo e produz resíduo ativo. $\mathrm{O}$ ativo é gerado nas atividades cotidianas de ensino e de pesquisa.

A elaboração de um programa de gerenciamento de resíduos requer mudança de atitudes e realimentação contínua. Por este motivo é importante que seja bem discutido e executado pelos responsáveis pela manutenção do mesmo. É importante conscientizar a comunidade universitária - professores, servidores técnico-administra- tivos e estudantes - a respeito da importância da disposição final dos resíduos perigosos oriundos das aulas e pesquisas laboratoriais. Este trabalho é uma extensão natural das atividades deste projeto e estima-se que tenha um impacto positivo, contribuindo para diminuir riscos e minimizar a insalubridade no local de trabalho.

\section{Redução do consumo e reutilização de descartáveis}

Em parceria com a direção da FFP/UERJ pretendemos distribuir e estimular o uso da caneca individual, além da instalação de coletores de copos descartáveis, visando a sua reutilização como recipiente na produção de mudas de plantas.

\section{Reflorestamento}

Na produção de mudas para o reflorestamento serão selecionadas, prioritariamente, espécies frutíferas da Mata Atlântica, que servirão não só para atração da avifauna ${ }^{28}$, mas, para ampliar a relação homem-planta dentro do campus da FFP/UERJ. Isso acontecerá nas épocas de frutificação, onde, com certeza as comunidades acadêmica e vizinha serão atraídas pelos frutos. Com essa medida pretende-se mitigar o efeito "Plant Blindness", ou seja, a tendência que a maioria das pessoas tem de ignorar os vegetais em detrimento dos animais ${ }^{29}$. A lista de espécies será elaborada através de consulta a fontes bibliográficas ${ }^{30,31} \mathrm{e}$ as mudas serão produzidas por sementes ou estaquia. Dar-se-á preferência para a reutilização de copos descartáveis utilizados na FFP/UERJ e garrafas PET para a produção de recipientes individuais. Parte do substrato utilizado para preencher os recipientes e as sementeiras virá da composteira instalada no reflorestamento da FFP/UERJ. O envolvimento da comunidade interna no reflorestamento do campus e adjacências será estimulado através de ações do tipo, "cada calouro uma muda" em que os ingressantes serão responsáveis pelo plantio e acompanhamento do desenvolvimento das plantas no reflorestamento.

Esta ação visa dar continuidade ao reflorestamento iniciado na FFP/UERJ em 1999, em uma área com aproximadamente $500 \mathrm{~m}^{2}$, onde foram plantados aproximadamente 178 indivíduos de 27 espécies nativas da Mata Atlântica, doadas pelo Instituto de Pesquisas Jardim Botâni- 
co do Rio de Janeiro, o antigo Instituto Estadual de Florestas, alunos e professores da FFP/UERJ ${ }^{8}$. Atualmente é realizada a manutenção do local, com reposição das mudas que foram cortadas ou sofreram ação do fogo.

Atualmente está em construção a proposta de oficinas de ensino ligadas ao Núcleo de Pesquisa e Ensino de Ciências da FFP (NUPEC/FFP), que envolve atividades de identificação das árvores plantadas, relacionando-as com a ecologia local, a montagem das cadeias tróficas em associação com as informações sobre as aves, adaptações para dispersão de sementes e frutos, polinização e competição por recursos. Pretende-se também ministrar palestras sobre a recuperação de áreas degradadas, o problema das espécies exóticas invasoras, a inibição do processo de recuperação pelo fogo, o grau de compactação do solo e serrapilheira, entre outros fatores bióticos e abióticos. Esse subprojeto, capitaneado pelo Grupo de Estudos Interdisciplinares do Ambiente (GEIA), será realizado em parceria com escolas públicas da região. Discentes da FFP/UERJ orientarão as atividades e os resultados serão discutidos de forma a estabelecer uma metodologia de Educação Ambiental em áreas degradadas. Dessa maneira, espera-se a sensibilização para as questões ambientais locais, pressuposto estabelecido como de vital importância nas convenções internacionais e nacionais sobre o tema ${ }^{3,32}$.

\section{Produção de vídeo}

Com o apoio técnico do laboratório de produção de vídeos do NUPEC, será elaborado um roteiro e um vídeo visando à sensibilização da comunidade acadêmica contra o desperdício e estimulando a redução, reutilização e reciclagem do lixo. O vídeo será exibido como uma das atividades de "boas-vindas" aos calouros e a toda comunidade da FFP/UERJ.

\section{Conclusões}

A FFP/UERJ é uma unidade por onde circulam diariamente cerca de 3000 pessoas, que geram resíduos e têm a percepção ambiental da necessidade de mudanças no seu entorno. Entendendo que a Universidade tem o papel social de fomentar o debate e propor estratégias que permitam aproximar a comunidade destas questões, este projeto procurou reunir iniciativas que vislumbrassem estas demandas e se entrelaçassem a partir dos resíduos produzidos.

Com esse pensamento integrador, o projeto vem propondo atividades de redução, reutilização ou reciclagem de resíduos. Os resíduos orgânicos serão reciclados através da compostagem, o composto gerado utilizado na produção de mudas de plantas para o reflorestamento da FFP/UERJ, as árvores atrairão um número maior de aves e estas poderão ser observadas em atividades de Educação Ambiental.

\section{Agradecimentos}

A SR3/UERJ pelas bolsas concedidas aos alunos que atuam neste projeto. A direção da FFP/ UERJ pelo apoio e aos funcionários que responderam aos questionários. A FAPERJ pelos recursos financeiros utilizados neste trabalho, concedidos ao Prof. Dr. Ricardo T. Santori, através do edital EXTPESQ 2010 (proc. FAPERJ 111151/2010). A SR2 pela bolsa do PROGRAMA PROCIÊNCIA concedida aos Profs. Marcelo Guerra Santos e Ricardo T. Santori.

\section{Contribuição dos autores}

M. G. Santos foi responsável principal pela análise e interpretação dos dados, redação do artigo e aprovação final da versão a ser publicada.

G. R. da Silva, M. L. C. Azevedo, M. M. Barcellos, M. M. Sérgio, R. de F. Souza, A. A. M. de Barros, D. de S. Pimentel e L. J. S. Pinto participaram do desenvolvimento do projeto, da análise e interpretação dos dados.

M. C. F. dos Santos, R. R. L. Mendes e R. T. Santori colaboraram na análise e interpretação dos dados, na revisão crítica do texto e aprovação da versão final do artigo a ser publicado.

\section{Referências}

1. PENATTI, Fábio Eduardo; GUIMARÃES, S.T.L.; SILVA, P.M. da. Gerenciamento de resíduos químicos em laboratórios de análises e pesquisa: o desenvolvimento do sistema em laboratórios da área química. In: Workshop Internacional em Indicadores de Sustentabilidade - WIPIS II. São Carlos. 2008.

2. FIGUEIREDO, Paulo Jorge Moraes. A sociedade do lixo, os resíduos, a questão energética e a crise ambiental. 2. ed. Piracicaba: UNIMEP, 1995. 240 p. 
3. DIAS, Genebaldo Freire. Educação ambiental: princípios e práticas. São Paulo: Ed. Gaia. 2003. 551 p.

4. PELIZZOLI, Marcelo Luiz. A emergência do paradigma ecológico: reflexões ético-filosóficas para o século XXI. Petrópolis: Ed. Vozes, 1999. 160 p.

5. LEONARDI, Maria Lúcia Azevedo. Educação Ambiental e teorias econômicas: primeiras aproximações. In: ROMEIRO, A.R.; REYDON, B.P.; LEONARDI, M.L.A. (Org.) Economia do meio ambiente: teoria, políticas e a gestão de espaços regionais. Campinas: Unicamp Ed. 1996. p. 240-262.

6. LOUREIRO, Carlos Frederico Bernardo; AZAZIEL, Marcus; FRANCA, Nahyda. Educação ambiental e gestão participativa em unidades de conservação. 2. ed., Rio de Janeiro: IBAMA/IBASE. 2005, 43 p.

7. LOUREIRO, Carlos Frederico Bernardo. Educação ambiental e gestão participativa na explicitação e resolução de conflitos. Gestão em Ação, v. 7, n. 1, p. 1-16. 2004.

8. PIMENTEL, Douglas de Souza; SANTOS, Maria Cristina Ferreira dos; LEMOS, Glauber de Almeida; et al. Programa de recuperação ambiental do entorno da Faculdade de Formação de Professores, UERJ/São Gonçalo, RJ. Revista Interagir: pensando a extensão, Rio de Janeiro, n. 1, p. 25-28, 2001.

9. BRAGA, Maria Neuma Carvalho. O Município de São Gonçalo e sua História. 3. ed. Niterói: Ntpress, 2006. 376 p.

10. SOUSA, C.L.M.T. A formação do bairro Paraíso / Patronato no Município de São Gonçalo. São Gonçalo: Monografia do Departamento de Geografia, Faculdade de Formação de Professores da UERJ, 1999. 43 p.

11. TAVARES, Maria Tereza Goudart; SILVA, Catia Antonia da. Catálogo-observatório acadêmico-institucional da Faculdade de Formação de Professores. Rio de Janeiro: UERJ/FFP, 2010. 24 p.

12. OlIVEIRA, S. A.; LEITE, V. D.; PRASAD, S.; et al. Estudo da produção per capita de resíduos sólidos domiciliares da cidade de Campina Grande - PB. Revista Saúde e Ambiente, v. 5, n. 2, p. 37-44. 2004.

13. FERREZ, Lilla. Observando Aves no Estado do Rio de Janeiro. Contagem: Littera Maciel, 1992. 124 p.

14. HOFling, Elizabeth; CAMARGO, Hélio. Aves no Campus. 3. ed. São Paulo: EDUSP, 2002. 157 p.

15. REINERT, Bianca Luiza; BORNSCHEIN, Marcos Ricardo; BELMONTE-LOPES, Ricardo. Conhecendo aves silvestres brasileiras. Londrina: Vila Verde, 2004. 176 p.

16. SOUZA, Deodato. Todas as aves do Brasil. Guia de campo para identificação, 2. ed. Feira de Santana: Dall, 2004. 350 p.

17. FRISCH, Johan Dalgas; FRISCH, Christian Dalgas. Aves brasileiras e plantas que as atraem. 3. ed. São Paulo: Ed. Dalgas Ecoltec, 2005. 476 p.

18. SIGRIST, Tomas. Guia de campo - Aves do Brasil Oriental. São Paulo: Avis Brasilis, 2007. 448 p.

19. PEREIRA, José Felipe Monteiro. Aves e pássaros comuns do Rio de Janeiro. Rio de Janeiro: Technical Books Editora, 2008. 164 p.

20. RUBERG, Claudia; NEUFELD, Ângela Denise Hubert; GONÇALVES, Rosangela Silva; et al. Resíduos sólidos gerados na Universidade Federal do PAMPA - Campus São Gabriel/RS: Estimando a geração per capita. In: Congresso Brasileiro de Engenharia Sanitária e Ambiental, 25. Recife-PE, Associação Brasileira de Engenharia Sanitária e Ambiental, 25. 2009.

21. INSTITUTO BRASILEIRO DE GEOGRAFIA E ESTATÍSTICA. Censo demográfico 2000: características da população e dos domicílios - resultados do universo. Rio de Janeiro: IBGE, 2000. 550 p.

22. INSTITUTO BRASILEIRO DE GEOGRAFIA E ESTATÍSTICA. Pesquisa Nacional de Saneamento Básico. 2000. Rio de Janeiro: IBGE, 2002. 397 p.

23. PIMENTEL, Douglas de Souza; LEMOS, A.G.; MOTTA, Ellen Serri et al. Educação Ambiental em áreas carentes: uma experiência no Município de São Gonçalo, Rio de Janeiro, Brasil. Discursos: língua, cultura e sociedade. Número especial: Tendências atuais em Educação Ambiental. Lisboa: Universidade Aberta. p. 317-327. 2004.

24. RAPOSO, Alexandre; ORMOND, Luciana C.; RESEN DE, Tatiana; et al. Desenvolvendo projetos no ensino de zoologia: estudo preliminar da avifauna do campus da Faculdade de Formação de Professores da UERJ. In: Encontro Regional de Ensino de Biologia RJ/ES, II. São Gonçalo: Sociedade Brasileira de Ensino de Biologia, p. 214-217. 2003.

25. SILVEIRA, Aline Barbosa; CORRÊA, Fernanda Simas; RIBEIRO, Flávia Barata; et al. As aves do campus da Faculdade de Formação de Professores da UERJ (São Gonçalo, RJ) e sua percepção pela comunidade. Vozes em Diálogo. v. 1, n. 1, p. 100-108. 2005.

26. SILVA, M. B.; MAMEDE, Simone Batista. Grupos de observadores de aves e mamíferos como estratégia para a conservação da biodiversidade do Cerrado. In: Congresso regional de educação ambiental para a conservação do Cerrado, I. Quirinópolis-Goiás. p. 55-58. 2005.

27. JARDIM, Wilson de Figueiredo. Gerenciamento de Resíduos Químicos em Laboratórios de Ensino e Pesquisa. Química Nova, v. 21, n. 5, p. 671-673. 1998.

28. SANCHOTENE, Maria do Carmo Conceição. Frutíferas Nativas úteis à fauna na arborização urbana. 2. ed. Porto Alegre: SAGRA, 1989. 306 p.

29. WANDERSEEM, James H.; SCHUSSLER, Elisabeth E. Toward a Theory of Plant Blindness. Plant Science Bulletin, v. 47, n. 1, p. 2-9. 2001

30. LORENZI, Harri. Árvores Brasileiras: manual de identificação e cultivo de plantas arbóreas nativas do Brasil. Nova Odessa: Editora Plantarum, 1992. 352 p.

31. SILVA, Silvestre; TASSARA, H. Frutas no Brasil. São Paulo: Empresa das Artes e Edições Artísticas Ltda, 1996. $233 \mathrm{p}$.

32. UNESCO. Educação ambiental: as grandes orientações da Conferência de Tbilisi. Brasília: Instituto Brasileiro de Meio Ambiente e Recursos Naturais Renováveis, 1998. $154 \mathrm{p}$. 


\section{Abstract}

This paper aims to conduct a qualitative and quantitative analysis of residues produced in Teachers College (FFP) of the Rio de Janeiro State University (UERJ); diagnose of residues management and proposal of mitigation measures and activities of extension of environmental with schools. The diagnosis of residues management was conducted through structured interviews and qualitative and quantitative analysis by means of weighing and visual identification of waste for five weeks. As educational measure, we realized birdwatching activities in the reforestation area of FFP to sensitize people about environmental conservancy needs. Quantitative analysis showed a per capita production of solid waste of $26.46 \mathrm{~g}$ / day per user. The results indicated that there is the need for a more integrative waste management policy and that it is not just about cleaning the premises of the local. The present mitigating actions are: the reuse of paper in notebooks production, composting vegetal residues and recycling of used cooking oil at the canteen to produce soap. In the reforestation area in FFP 41 species of birds have already been observed. The future actions include development of a chemical waste management plan, reduction of recyclables, reforestation and production of a themed video.

Keywords: Educational Action; Residues Management; Reforestation; Birdwatching 